\title{
Propiedad industrial y licenciamiento en los Centros Conacyt
}

\author{
Industrial property and licensing in the Conacyt Centers
}

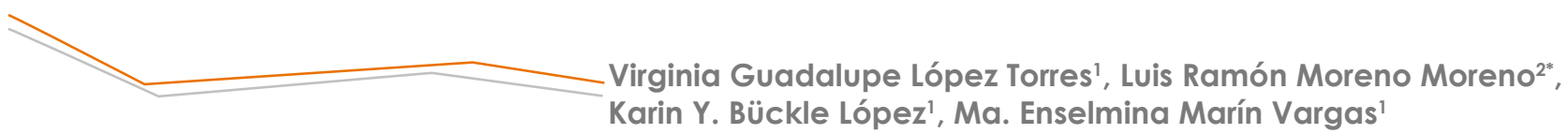

López Torres, V. G. Moreno Moreno, L. R. Bückle López, K. Y. Marín Vargas, M. E. Propiedad industrial y licenciamiento en los Centros Conacyt. Investigación y Ciencia de la Universidad Autónoma de Aguascalientes. Número 69: 54-63, septiembre-diciembre 2016.

\section{RESUMEN}

El estudio tiene como objetivo documentar el estado que guarda la propiedad industrial de los Centros Conacył y su transferencia. La investigación se basa en un estudio realizado a 18 centros públicos de investigación Conacyt, con información obtenida mediante la aplicación de un cuestionario, así como los informes de rendición de cuentas de cada uno de los 18 centros estudiados. Los resultados indican que $30 \%$ de las patentes, modelos de utilidad y diseños industriales con registro en el extranjero se han transferido a los sectores productivos, mientras que las patentes con registro nacional solamente se han transferido en $9 \%$ de los casos. En lo que respecta a los modelos de utilidad y diseños industriales con registro nacional, no se obtuvo información de que estos hayan sido transferidos. Esto representa una oportunidad para compartir conocimiento y generar ventajas competitivas.

\section{ABSTRACT}

The study aims to document the status of the industrial property and transfer strategies of Conacyt Centers. The research is based on a study of 18 Conacyt public

Palabras clave: Centros Conacyt, innovación, propiedad industrial, patentes, transferencia, licencias.

Keywords: Conacyt Centers, innovation, industrial property patents, transfer, licenses.

Recibido: 25 de agosto de 2015, aceptado: 14 de junio de 2016

Universidad Autónoma de Baja California, Facultad de Ciencias Administrativas y Sociales.

2 Universidad Autónoma de Baja California, Facultad de Ciencias Administrativas.

* Autor para correspondencia: nomarsiul@gmail.com research centers with information obtained through a questionnaire and the annual reports of each of the 18 studied centers. The results indicate that $30 \%$ of patents, utility models and industrial designs are registered abroad and have been transferred to the productive sectors, while patents with national registration have been transferred only in $9 \%$ of cases. With respect to utility models and industrial designs with national registration, no information was obtained that confirms that these have been transferred. This represents an opportunity to share knowledge and build competitive advantage.

\section{INTRODUCCIÓN}

La medición del esfuerzo que realiza un país en ciencia, tecnología e innovación (CTI) está representado por el gasto en Investigación y Desarrollo Experimental (IDE) con respecto a su Producto Interno Bruto (PIB). En México, $50 \%$ del gasto IDE/PIB es realizado por el gobierno a través de distintos agentes (Conacyt, 2008), principalmente universidades y centros de investigación, cuya misión es producir conocimientos que permitan solucionar problemas y demandas del sector empresarial y de la sociedad en general (López et al., 2006).

Cuando se habla de innovación desde una perspectiva de desarrollo económico y empresarial, el Manual de Oslo 2005 la reconoce como "la puesta en práctica de un nuevo o significativamente mejorado producto (bien o servicio), un nuevo proceso, un nuevo método de comercialización, o un nuevo método de: prácticas comerciales, organización del lugar de trabajo, o relaciones exteriores" (OCDE y Eurostat, 2005: 24). 
Cabe destacar que para que la innovación (o sus procesos) se tornen productivos, es necesaria la aplicación de la misma; al respecto Kantis y Díaz (2008: 11), plantean que la comercialización de las innovaciones se realiza de dos formas: "Por un lado, está la transferencia de derechos de patentamiento - licencias a una empresa para que la lleve al mercado. Otra forma es a través de la creación de una empresa que nace con el propósito de explotarla".

Los resultados del Índice Global de Innovación 2013, editado por Dutta y Lanvin (2013) ilustran un avance de México al ubicarse en la posición 63 (de un total de 142 economías analizadas) cuando en el año 2012 se ubicó en la posición 79; es decir, se observa una mejoría importante en las calificaciones de distintos subíndices con un avance de 16 posiciones. Es pertinente indicar que el Índice Global de Innovación se construye a partir de dos subíndices: entradas de innovación y salidas de innovación, los cuales a su vez se determinan con una serie de variables denominadas pilares.

En el caso específico de México, si bien se presenta una mejora en lo general, en el pilar denominado sofisticación del negocio, el país se ubica en la posición 89 en el año 2013 (caída en dos lugares con respecto a 2012), lo que muestra un panorama crítico, con considerables retos para los subpilares: empleo intensivo en conocimiento (lugar 92) y vinculaciones de innovación (lugar 98). El primero mide, entre otros indicadores, la variable de investigación y desarrollo (I\&D) en las empresas y la capacidad emprendedora; mientras que el segundo documenta las colaboraciones de I\&D universidad-empresa y el número de alianzas estratégicas, que cabe destacar, son los indicadores vinculados directamente con el análisis del presente documento (Cámara de Diputados, 2014).

En México, de acuerdo con los artículos 1 y 2 de la Ley Orgánica del Consejo Nacional de Ciencia y Tecnología (Cámara de Diputados, 2014), el Conacył es el organismo responsable de elaborar las políticas públicas relacionadas con la ciencia y la tecnología; asimismo, debe impulsar tareas de investigación a través del Sistema de Centros de Investigación (SCl), el cual está integrado por 27 instituciones (en adelante Centros Conacyt (CC)) que cubren los principales campos del conocimiento científico y tecnológico. Entre los objetivos que persiguen los CC destacan fomentar la tecnología local generando los mismos resultados que la tecnología extranjera; innovar en la generación, desarrollo, asimilación y aplicación del conocimiento y vincular la ciencia y tecnología con la sociedad y el sector productivo para atender sus problemas (Conacyt, 2014).

Es pertinente señalar que en las evaluaciones internacionales asociadas a procesos educativos (como PISA: Programa para la Evaluación Internacional de los Alumnos), de innovación (Índice Global de Innovación) y desempeño económico (Índice de Competitividad Global e Índice de Desempeño Logístico), generalmente la economía mexicana

Tabla 1. Gasto federal en ciencia y tecnología 2002-2011

\begin{tabular}{|c|c|c|c|c|c|}
\hline \multirow{2}{*}{ Año } & \multicolumn{2}{|c|}{ GFCyT } & \multicolumn{2}{c|}{ PIB } & \multirow{2}{*}{ GFCyT/PIB } \\
\cline { 2 - 5 } & $\begin{array}{c}\text { A precios } \\
\text { corrientes }\end{array}$ & $\begin{array}{c}\text { A precios de } \\
\mathbf{2 0 1 1}\end{array}$ & $\begin{array}{c}\text { A precios } \\
\text { corrientes }\end{array}$ & $\begin{array}{c}\text { A precios de } \\
\mathbf{2 0 1 1}\end{array}$ & \\
\hline 2002 & $24,363.9$ & $41,730.1$ & $6,815,546.5$ & $11,673,583.7$ & 0.36 \\
\hline 2003 & $29,309.0$ & $45,881.2$ & $7,555,803.4$ & $11,828,070.7$ & 0.39 \\
\hline 2004 & $27,952.1$ & $40,128.6$ & $8,574,823.2$ & $12,310,171.7$ & 0.33 \\
\hline 2005 & $31,339.0$ & $43,027.5$ & $9,251,737.5$ & $12,702,350.8$ & 0.34 \\
\hline 2006 & $33,275.8$ & $42,823.5$ & $10,379,091.0$ & $13,357,121.8$ & 0.32 \\
\hline 2007 & $35,831.7$ & $43,658.7$ & $11,320,836.4$ & $13,793,719.7$ & 0.32 \\
\hline 2008 & $43,829.2$ & $50,210.9$ & $12,181,256.1$ & $13,954,888.9$ & 0.36 \\
\hline 2009 & $45,973.6$ & $50,452.3$ & $11,923,678.8$ & $13,085,262.2$ & 0.39 \\
\hline 2010 & $54,436.4$ & $57,417.9$ & $13,089,273.1$ & $13,806,188.6$ & 0.42 \\
\hline 2011 & $58,809.88$ & $58,809.88$ & $14,352,866.79$ & $14,352,866.79$ & 0.41 \\
\hline
\end{tabular}


presenta resultados mediocres. Al respecto, autores como Dautrey (2008), Salvador Benítez (2008), Villalobos García y Ponce Talancón (2008), Lomelí Vanegas y Murayama Rendón (2009) y Cabello y Ortiz (2013) documentan que estos escasos resultados de desempeño son consecuencia de un insuficiente gasto público en procesos educativos y de investigación y desarrollo. En ese marco, por ejemplo, se observa que si bien es cierto que el gasto en la variable de ciencia y tecnología ha aumentado $141.3 \%$ en valores corrientes, la proporción de gasto en este rubro como proporción del PIB se ha mantenido más o menos constante (Tabla 1). A nivel internacional, estos valores están muy alejados de lo que se ejerce; por ejemplo, en Suecia y Japón, que son de los países que más invierten en ciencia y tecnología, con valores superiores a $3 \%$ del PIB (Tabla 2).

Tabla 2. Gasto en investigación y desarrollo por país, 2010

\begin{tabular}{|c|c|c|}
\hline País & $\begin{array}{c}\text { GIDE (Millones de } \\
\text { PPP corrientes }\end{array}$ & GIDE/PIB (\%) \\
\hline Alemania & $86,209.6$ & 2.82 \\
\hline EE. UU. (2009) & $401,576.0$ & 2.90 \\
\hline Francia & $49,990.8$ & 2.26 \\
\hline Japón (2009) & $137,314.2$ & 3.36 \\
\hline México & $7,876.0$ & 0.48 \\
\hline Suecia & $12,535.5$ & 3.43 \\
\hline
\end{tabular}

1/ La paridad del poder adquisitivo (PPP, por sus siglas en inglés) es la tasa de conversión de moneda que elimina las diferencias en niveles de precios entre países. Fuente: INEGI (s. f. a), OECD (2012).

En el entorno mundial, la oficina de registros de México participa con menos de $1 \%$ del total de registros anuales en el rubro de patentes (Tabla 3), la lista es encabezada por China con cerca de $28 \%$ del total. Habría que agregar que de acuerdo a los datos de la Organización Mundial de Propiedad Intelectual (WIPO, por sus siglas en inglés) (2013), alrededor de $92 \%$ de las patentes registradas en el país corresponden a no residentes del mismo. Estos datos reflejan que se deben desarrollar e impulsar las capacidades de innovación en el país.

De acuerdo a la WIPO (2013), la propiedad intelectual se divide en propiedad industrial $y$ derecho de autor, donde la propiedad industrial abarca las patentes de invención, las marcas, los diseños industriales y las indicaciones geográficas. Como se ha mencionado, existen cuatro formas de propiedad industrial, pero este artículo está centrado únicamente en las patentes y los modelos de utilidad que tienen la función de proteger las invenciones, y los diseños industriales, que tienen que ver con creaciones estéticas que son determinantes en el aspecto de los productos industriales.

Es importante señalar que en México la protección de la inventiva industrial se enmarca en la Ley de Fomento y Protección de la Propiedad Industrial (SECOFI, 27 de junio de 1991), la cual asegura los derechos patrimoniales al (los) inventor(es) y dependiendo de la forma de registro, también a la empresa u organización (titular) que haya impulsado o financiado la invención. Las invenciones se pueden proteger bajo cuatro figuras jurídicas: patentes, modelos de utilidad (MU), diseños industriales (DI) y trazado de circuitos.

El O los titulares de las invenciones tienen derecho de celebrar contratos de cesión o licencia para su uso, a fin de que la invención llegue al mercado e incluso propicie la creación de empresas tipo spin-off (es decir, aquellas entidades económicas creadas a partir de una innovación cuyos miembros fundadores suelen ser investigadores o personal de centros de investigación o universidades). Con ello el conocimiento explícito en la patente se disemina y usa. La patente proporciona amplia información del invento, por ello es considerada como un indicador confiable de la transferencia de conocimientos (OCDE, 2009).

En México, la propiedad industrial se registra en el Instituto Mexicano de Propiedad Industrial (IMPI). Este organismo emite un reporte que documenta las diferentes figuras jurídicas y el registro anual de patentes en el país. En ese marco, la Figura 1 presenta la evolución en los registros de patentes ante el IMPI para el periodo comprendido desde 1993 hasta 2010, de acuerdo a la nacionalidad del titular que somete la innovación para registro. Puede observarse que la tendencia de los titulares nacionales no presenta cambios significativos, lo confirma el discurso del director general de Conacyt, Dr. Enrique Villa, en el marco del Día Mundial de la Propiedad Intelectual (dictado en el Distrito Federal el 26 de abril de 2011), donde declara que el número de patentes que produce México es similar a las solicitadas por Francia; pero a diferencia de Francia, en México la mayoría de los solicitantes son extranjeros (Conacyt, 2011).

En el mismo tenor, la Ley de Ciencia y Tecnología (Cámara de Diputados, 2002) plantea 
Tabla 3. Aplicaciones de patentes por las 15 oficinas principales

\begin{tabular}{|l|c|c|c|c|}
\hline \multicolumn{1}{|c|}{ Oficina } & $\mathbf{2 0 0 8}$ & $\mathbf{2 0 1 0}$ & $\mathbf{2 0 1 2}$ & $\begin{array}{c}\text { Participación en total } \\
\mathbf{2 0 1 2}(\mathbf{\%})\end{array}$ \\
\hline Total & 1915000 & 1979000 & 2347700 & \\
\hline Estados Unidos de América & 456321 & 490226 & 542815 & 23.12 \\
\hline China & 289838 & 391177 & 652777 & 27.80 \\
\hline Japón & 391002 & 344598 & 342796 & 14.60 \\
\hline República de Corea & 170632 & 170101 & 188915 & 8.05 \\
\hline Oficina de Patentes Europea & 146150 & 150961 & 148560 & 6.33 \\
\hline Alemania & 62417 & 59245 & 61340 & 2.61 \\
\hline Federación Rusa & 41849 & 42500 & 44211 & 1.88 \\
\hline Canadá & 42089 & 35449 & 35242 & 1.50 \\
\hline Brasil & 22917 & 22686 & 30116 & 1.28 \\
\hline Reino Unido & 23379 & 21929 & 23235 & 0.99 \\
\hline Francia & 16419 & 16580 & 16632 & 0.71 \\
\hline México & 16581 & 14576 & 15314 & 0.65 \\
\hline Hong Kong, China & 13662 & 11702 & 12988 & 0.55 \\
\hline Otros & 158586 & 182383 & 162446 & 6.92 \\
\hline
\end{tabular}

Fuente: WIPO (2013).

entre sus objetivos vincular a los sectores educativo, productivo y de servicios en materia de investigación científica, desarrollo tecnológico e innovación.

El artículo 40 Bis precisa que entre otros, los CC podrán crear unidades de vinculación y transferencia de conocimiento. El artículo 51 establece además que los centros promoverán la conformación de asociaciones estratégicas, alianzas tecnológicas, consorcios, nuevas empresas privadas de base tecnológica y redes regionales de innovación en las

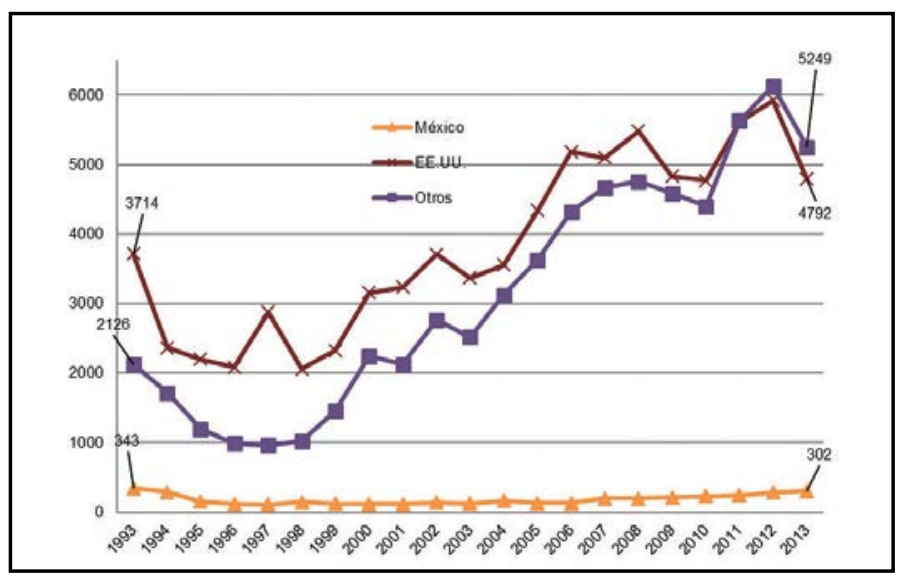

Figura 1. Tendencia en patentes registradas ante IMPI, 1993-2013. Elaboración propia con datos de IMPI (2014). cuales se procurará la incorporación de desarrollos tecnológicos e innovaciones realizadas en dichos centros, así como de los investigadores formados en ellos.

Por otra parte y de acuerdo al Conacyt (2014), los centros son el motor de la formación de recursos humanos, debido a que generan $75 \%$ de la actividad científica y tecnológica del país. Es importante y pertinente señalar que el Sistema de Centros de Investigación (SCl) se clasifica en tres subsistemas: agrupa $10 \mathrm{CC}$ en el subsistema de ciencias exactas y naturales (SCEyN), 8 en el subsistema de ciencias sociales y humanidades (SCSyH) y los restantes ocho en el subsistema de desarrollo e innovación tecnológica (SDIT).

Los llamados Indicadores Anuarios publicados por Conacyt ilustran los resultados de los CC y en ellos se pueden encontrar tres tipos de productos generados por estos: publicaciones, estudiantes graduados y patentes. Estas últimas constituyen el único indicador directo de innovación en materia industrial; los datos para el periodo comprendido desde 2001 hasta 2013 son los que se muestran en la Tabla 4 , donde destaca el hecho de que durante todo el periodo de análisis el número de patentes ascendió a 480, con una mayor participación de Corporación 
Tabla 4. CC y patentes registradas de 2001-2013

\begin{tabular}{|c|c|c|}
\hline Centro Conacyt (CC) & Subsistema & $\begin{array}{l}\text { Patentes } \\
\text { registradas } \\
2001-2013\end{array}$ \\
\hline $\begin{array}{l}\text { Centro de Investigación en } \\
\text { Alimentación y Desarrollo, } \\
\text { A.C. (CIAD) }\end{array}$ & \multirow{9}{*}{ SCEyN } & 8 \\
\hline $\begin{array}{l}\text { Centro de Investigaciones } \\
\text { Biológicas del Noroeste, S.C. } \\
\text { (ClBNOR) }\end{array}$ & & 8 \\
\hline $\begin{array}{l}\text { Centro de Investigación } \\
\text { Científica y de Educación } \\
\text { Superior de Ensenada, B.C. } \\
\text { (CICESE) }\end{array}$ & & 6 \\
\hline $\begin{array}{l}\text { Centro de Investigación } \\
\text { Científica de Yucatán, A.C. } \\
\text { (ClCY) }\end{array}$ & & 7 \\
\hline $\begin{array}{l}\text { Centro de Investigación en } \\
\text { Matemáticas, A.C. (CIMAT) }\end{array}$ & & 2 \\
\hline $\begin{array}{l}\text { Centro de Investigación en } \\
\text { Materiales Avanzados, S. C. } \\
\text { (CIMAV) }\end{array}$ & & 48 \\
\hline $\begin{array}{lcr}\text { Instituto } & \text { Nacional } & \text { de } \\
\text { Astrofísica, } & \text { Óptica } & \text { y } \\
\text { Electrónica (INAOE) } & \end{array}$ & & 21 \\
\hline $\begin{array}{l}\text { Instituto de Ecología, A. C. } \\
\text { (INECOL) }\end{array}$ & & 4 \\
\hline $\begin{array}{l}\text { Instituto Potosino de } \\
\text { Investigación } \\
\text { (IPICYT) }\end{array}$ & & 18 \\
\hline $\begin{array}{l}\text { Centro de Investigación en } \\
\text { Química Aplicada (CIQA) }\end{array}$ & \multirow{8}{*}{ SDIT } & 97 \\
\hline $\begin{array}{l}\text { Centro de Innovación } \\
\text { Aplicada en Tecnologías } \\
\text { Competitivas (CIATEC) }\end{array}$ & & 14 \\
\hline $\begin{array}{l}\text { Centro de Investigación y } \\
\text { Asistencia en Tecnología y } \\
\text { Diseño del Estado de Jalisco, } \\
\text { A.C. (CIATEJ) }\end{array}$ & & 60 \\
\hline $\begin{array}{l}\text { CIATEQ, A.C. Centro de } \\
\text { Tecnología } \\
\text { (CIATEQ) }\end{array}$ & & 16 \\
\hline $\begin{array}{l}\text { Centro de Ingeniería y } \\
\text { Desarrollo Industrial (CIDESI) }\end{array}$ & & 16 \\
\hline $\begin{array}{l}\text { Centro de Investigación } \\
\text { y Desarrollo Tecnológico } \\
\text { en Electroquímica, S. C. } \\
\text { (CIDETEQ) }\end{array}$ & & 11 \\
\hline $\begin{array}{l}\text { Corporación Mexicana de } \\
\text { Investigación en Materiales, } \\
\text { S. A. de C. V. (COMIMSA) }\end{array}$ & & 92 \\
\hline $\begin{array}{l}\text { Fondo de Información y } \\
\text { Documentación para la } \\
\text { Industria (INFOTEC) }\end{array}$ & & 4 \\
\hline
\end{tabular}

Adaptación a partir de Conacył (2000-2013).
Mexicana de Investigación en Materiales, S. A. de C. V. (COMIMSA), Centro de Investigación en Química Aplicada (CIQA) y Centro de Investigación y Asistencia en Tecnología y Diseño del Estado de Jalisco, A. C. (CIATEJ), que son CC pertenecientes al SDIT.

Sin embargo, De Gortari Rabiela (2002) señala la necesidad de que los CC adopten un enfoque hacia el mercado; es decir, el cambio de un esquema de technology push (empuje de tecnología) al de market pull (demanda del mercado), a fin de que puedan responder a los retos de la industria. En ese marco, se hace necesario un enfoque donde los investigadores incorporen capacidades de gestión y comercialización que permitan resolver problemas de la industria/empresa.

El objetivo del artículo es documentar el estado que guarda el registro de invenciones de los CC y su transferencia a empresas mexicanas por comercialización vía licencia o cesión. Para ello se describe el método de investigación; se presentan los resultados de esta investigación y, por último, las consideraciones finales.

\section{MATERIALES Y MÉTODOS}

Para la recolección de información se diseñó un instrumento tipo encuesta compuesto por cuatro categorías, que a su vez encierran un total de 12 preguntas. La información se obtuvo por dos vías: a) correo electrónico dirigido a los directivos de vinculación, comercialización y/o innovación de los CC, con solicitud, justificación e instrucciones para llenar la encuesta en línea y b) solicitudes de llenado de la encuesta usando los trámites de acceso a la información pública en el sitio electrónico del Instituto Federal de Acceso a la Información y Protección de Datos (IFAI).

El cuestionario buscaba obtener datos sobre el desarrollo de patentes que los CC (o sus investigadores) han registrado ante el IMPI y en el extranjero. Se les preguntó cuántas patentes, modelos de utilidad y diseños industriales conservan en modalidad de título otorgado para el periodo 2000-2013; se descartaron aquellos que estuvieran en proceso de solicitud. De la misma forma se solicitó información acerca de cuántos de esos títulos se habían transferido a empresas mexicanas y/o extranjeras.

La encuesta se enfocó a los CC del subsistema ciencias exactas y naturales (SCEyN) y subsistema de 
desarrollo e innovación tecnológica (SDIT), entonces 19 son sujetos de estudio; la tasa de respuesta fue de 18 CC (que corresponde a 95\%). Cabe destacar que 12 respondieron vía electrónica (encuesta en línea) y los restantes 6 a través de IFAI.

En ese marco, como ya se mencionó, el objetivo del presente trabajo es documentar el estado que guarda el registro de invenciones de los CC y su transferencia a empresas mexicanas; para ello se planteó el supuesto de que $30 \%$ de las invenciones protegidas bajo las figuras de patentes, modelos de utilidad y diseños industriales se ha transferido hacia el sector empresarial.

\section{RESULTADOS}

Los CC encuestados reportaron 306 títulos de patentes otorgados por el IMPI y 42 patentes registradas en el extranjero durante el periodo 2000 a 2013. Es decir, un total de 348 títulos de patentes. Por ello, existe una diferencia en los datos reportados en la encuesta y los datos reportados en los indicadores anuarios presentados en la Tabla 4. Para valorar esta diferencia se declara la siguiente hipótesis nula: $\mathrm{H}_{0}$ : No existe una diferencia significativa entre los datos de los indicadores anuarios de Conacyt y los datos de la encuesta. La hipótesis se probó utilizando la prueba ji cuadrada no paramétrica $\left(\chi^{2}\right)$, cuyo resultado es $\chi^{2}=$ 1.25761E-48, lo cual indica que se acepta la hipótesis nula. Por tanto, se infiere que no existe una diferencia significativa entre los datos reportados por Conacyt y los obtenidos mediante la encuesta.

De las 306 patentes registradas en IMPI, los CC reportan que 27 han sido transferidas a empresas nacionales (Tabla 5), donde destaca el Centro de Investigación en Materiales Avanzados, S. C. (CIMAV), mismo que ha transferido seis patentes; asimismo, la Corporación Mexicana de Investigación en Materiales, S. A. de C. V. (COMIMSA) y el Centro de Investigación en Química Aplicada (CIQA), ambos con cinco patentes transferidas. Esto implica que solamente $8.82 \%$ de los títulos de patente terminan en una licencia. Cabe mencionar que las patentes registradas en el extranjero tienen un comportamiento algo diferente; por ejemplo, 6 de las 36 patentes han sido transferidas en el extranjero; es decir, aproximadamente $17 \%$ de los títulos de patente terminan en una licencia.
Tabla 5. Patentes transferidas (2000-2013) por los CC en México

\begin{tabular}{|l|c|c|c|}
\hline CC & FE & FO & $\begin{array}{c}\text { Patentes } \\
\text { transferidas }\end{array}$ \\
\hline CIAD & 8 & 10 & 0 \\
\hline CIBNOR & 8 & 8 & 0 \\
\hline CICESE & 6 & 5 & 1 \\
\hline CICY & 7 & 6 & 3 \\
\hline CIMAT & 2 & 0 & 0 \\
\hline CIMAV & 48 & 25 & 6 \\
\hline CIO & 48 & 8 & 1 \\
\hline INAOE & 21 & 10 & 1 \\
\hline INECOL & 4 & 4 & 0 \\
\hline IPICYT & 18 & 3 & 0 \\
\hline CIATEC & 14 & 6 & 3 \\
\hline CIATEJ & 60 & 14 & 2 \\
\hline CIATEQ & 16 & 53 & 0 \\
\hline CIDESI & 16 & 4 & 0 \\
\hline CIDETEQ & 11 & 4 & 0 \\
\hline CIQA & 97 & 127 & 5 \\
\hline COMIMSA & 92 & 19 & 5 \\
\hline INFOTEC & 4 & 0 & 0 \\
\hline TOTAL & 480 & 306 & 27 \\
\hline
\end{tabular}

Elaboración propia con datos de la encuesta.

En relación con los modelos de utilidad, los CC reportaron 9 títulos registrados, de los cuales 4 se han transferido en México; de la misma forma, es importante señalar que solo siete CC producen este tipo de invención. Respecto a los diseños industriales, los CC reportan 58 títulos registrados, de los cuales 24 se han trasferido en México, con un porcentaje de transferencia de $41.37 \%$. Solamente cuatro de estos centros generan este tipo de invención (Tabla 6). Según el objetivo de los CC que hace alusión a la tarea de vincular la ciencia y tecnología con la sociedad y el sector productivo para atender sus

Tabla 6. Modelos de utilidad (MU) y Diseños industriales (DI) registrados y transferidos por los CC en México de 2000 a 2013

\begin{tabular}{|l|c|c|c|c|}
\hline CC & MU & $\begin{array}{c}\text { MU } \\
\text { transferidos }\end{array}$ & DI & $\begin{array}{c}\text { DI } \\
\text { transferidos }\end{array}$ \\
\hline CIBNOR & 1 & 1 & 0 & 0 \\
\hline CICY & 1 & 1 & 1 & 1 \\
\hline CIATEC & 1 & 1 & 19 & 18 \\
\hline CIATEJ & 1 & 1 & 0 & 0 \\
\hline CIDESI & 1 & 0 & 0 & 0 \\
\hline CIDETEQ & 1 & 1 & 1 & 0 \\
\hline COMIMSA & 3 & 0 & 37 & 5 \\
\hline TOTAL & 9 & 4 & 58 & 24 \\
\hline
\end{tabular}

Elaboración propia con datos de la encuesta. 
Tabla 7. Resultados obtenidos al aplicar las pruebas de hipótesis Ho: $p \leq 0.30$

\begin{tabular}{|l|c|l|}
\hline \multicolumn{1}{|c|}{ Concepto } & Estadístico & \multicolumn{1}{|c|}{ Inferencia } \\
\hline Patentes (registro nacional) & $\mathrm{z}=1.97$ & $\begin{array}{l}\text { Se acepta Ho, las patentes con registro nacional transferidas es } \\
\text { menor de 30\%. }\end{array}$ \\
\hline Patentes (registro en el extranjero) & $\mathrm{z}=1.16$ & $\begin{array}{l}\text { Se rechaza Ho, las patentes con registro en el extranjero } \\
\text { transferidas son mayores a 30\%. }\end{array}$ \\
\hline Modelos de utilidad & $\mathrm{z}=-1.25$ & $\begin{array}{l}\text { Se rechaza Ho, los modelos de utilidad transferidos son mayores } \\
\text { a 30\%. }\end{array}$ \\
\hline Diseños industriales & $\mathrm{z}=-0.98$ & $\begin{array}{l}\text { Se rechaza Ho, los diseños industriales transferidos son mayores } \\
\text { a 30\%. }\end{array}$ \\
\hline
\end{tabular}

Elaboración propia.

problemas y los resultados ilustrados, se infiere que el Centro de Innovación aplicada en Tecnologías Competitivas (CIATEC) es un centro bastante productivo y altamente vinculado, ya que ha transferido $84.61 \%$ de sus invenciones.

En relación con el supuesto planteado Ho (hipótesis nula): $30 \%$ de las invenciones de los CC protegidas bajo las figuras de patentes, modelos de utilidad (MU) y diseños industriales (DI), se ha transferido. Se valida con la prueba estadística de proporciones, con un nivel de confianza $\alpha=0.05$; los datos y cálculos se ilustran en la Tabla 7.

Por otro lado, de acuerdo con la información obtenida, no se observa una tendencia clara de que el monto de recursos financieros destinados a los distintos CC impliquen una mayor cantidad de patentes, o que de la misma forma, el número de investigadores tenga un impacto directo en esta variable. Por ejemplo, si uno toma en cuenta la información que aparece en la Tabla 8 (patentes, financiamiento, investigadores promedio y la proporción de investigadores miembros del Sistema Nacional de Investigadores con respecto al número total de investigadores), es posible observar que el Centro de Investigación en Materiales Avanzados, S. C. (CIMAV) es el que ocupa el primer lugar en número de patentes registradas para el periodo 2005-2008; sin embargo, ocupa la novena posición en términos de los recursos financieros destinados al mismo y la posición 17 de acuerdo a la cantidad de investigadores adscrita al centro. Cabe destacar, sin embargo, que ocupa la primera posición en cuanto a la proporción de investigadores clasificados dentro del Sistema Nacional de Investigadores (SNI) con respecto al total de investigadores de los centros.

Una situación similar a la anterior se presenta en el Centro de Investigaciones en Óptica, A. C. (CIO), que ocupa la segunda posición en términos de la cantidad de xpatentes generadas para el periodo de análisis y la décima posición en lo que respecta al financiamiento. En términos del número de investigadores promedio, ocupa la antepenúltima posición y el segundo lugar en la proporción de investigadores que cuentan con el reconocimiento del SNI.

Tabla 8. Patentes, financiamiento, \# de investigadores y relación entre los mismos e inversión total con el fin de identificar relaciones entre variables y los CC, 2005-2008

\begin{tabular}{|l|c|c|c|c|}
\hline \multicolumn{1}{|c|}{ Centro } & Patentes & Financiamiento & $\begin{array}{c}\text { Investigadores } \\
\text { promedio }\end{array}$ & $\begin{array}{c}\text { Inv. SNI/Inv. } \\
\text { Totales }\end{array}$ \\
\hline CIMAV & 34 & $467,380,206.0$ & 38 & $98.7 \%$ \\
\hline CIO & 29 & $448,270,369.0$ & 61 & $95.5 \%$ \\
\hline COMIMSA & 20 & $1,525,842,000.0$ & 183 & $1.8 \%$ \\
\hline CIQA & 19 & $489,983,885.0$ & 72 & $49.8 \%$ \\
\hline CIATEQ & 12 & $1,896,991,263.0$ & 190 & $5.9 \%$ \\
\hline CIATEJ & 11 & $385,181,025.0$ & 88 & $23.0 \%$ \\
\hline CIDESI & 11 & $798,578,763.0$ & 62 & $9.2 \%$ \\
\hline IPICYT & 11 & $420,667,441.0$ & 47 & $116.1 \%$ \\
\hline INAOE & 6 & $882,688,169.0$ & 129 & $78.4 \%$ \\
\hline CIATEC & 4 & $442,395,747.0$ & 92 & $10.3 \%$ \\
\hline CICESE & 2 & $1,368,924,866.0$ & 177 & $80.2 \%$ \\
\hline CIAD & 2 & $101,583,215.0$ & 175 & $44.6 \%$ \\
\hline CIBNOR & 2 & $1,164,778,161.0$ & 116 & $86.0 \%$ \\
\hline CIDETEQ & 2 & $276,263,747.0$ & 70 & $18.6 \%$ \\
\hline CIMAT & 1 & $492,658,694.0$ & 67 & $72.5 \%$ \\
\hline CICY & 1 & $18,392,428.0$ & 66 & $90.2 \%$ \\
\hline INECOL & 0 & $125,815,600.0$ & 110 & $59.2 \%$ \\
\hline
\end{tabular}

Elaboración propia con datos de Conacył (s. f.). 
Un caso interesante es el de Corporación Mexicana de Investigación en Materiales, S. A. de C. V. (COMIMSA), organización que ocupa la tercera posición en términos de la cantidad de patentes generadas en el periodo de análisis. Este centro ocupa el segundo lugar en términos del financiamiento recibido durante el periodo, así como el segundo lugar en cuanto al número de investigadores adscritos a esta institución. Lo interesante es que de sus investigadores solamente $1.8 \%$ cuentan con el reconocimiento del SNI.

\section{DISCUSIÓN}

A pesar de los hallazgos de la Tabla 8, los resultados deben tomarse bajo ciertas consideraciones, la principal que la inversión en ciencia y tecnología generalmente tarda años en rendir frutos debido a la naturaleza de los procesos de experimentación. En ese marco, las patentes mostradas en cuadros previos pueden ser el resultado de una transición hacia el desarrollo de una cultura de generación de resultados de innovación, de manera que aquellos centros que actualmente pueden no estar registrando patentes, pero sí están recibiendo financiamiento, tal vez lo hagan en el mediano plazo.

Asimismo, de acuerdo con Calderón Martínez (2014: 40), las pocas patentes transferidas

se vinculan con la baja capacidad de absorción por parte de la industria, las diferencias entre los resultados de investigación y las necesidades del sector productivo, así como con la debilidad de los incentivos existentes para la protección de la propiedad intelectual.

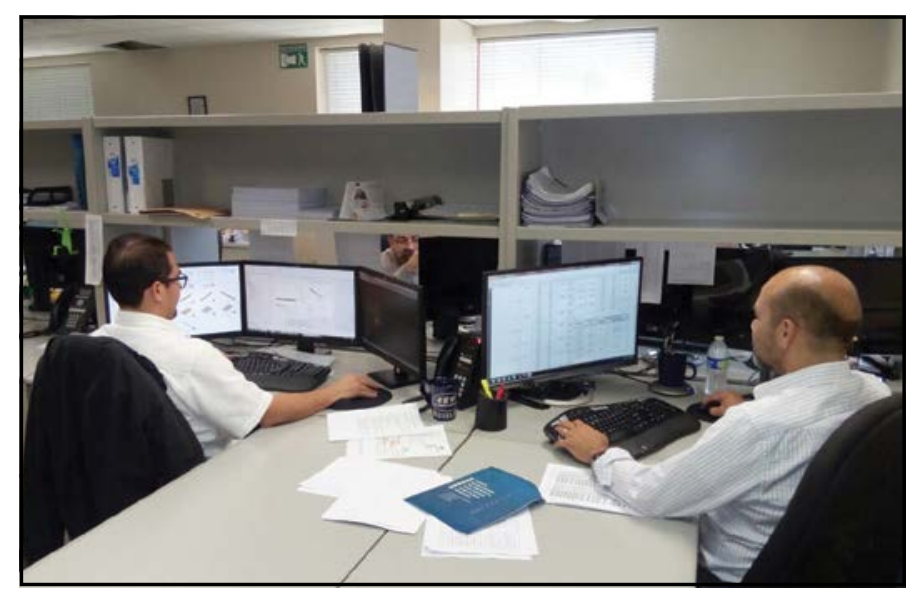

Figura 2. Se estudia la transferencia de propiedad industrial por comercialización.
En el mismo sentido, Closs et al. (2012) señalan que en Brasil el sector productivo no invierte en el desarrollo de nuevos productos, por lo que su capacidad de absorción de patentes es nula; indican además que no existe una cultura empresarial en las universidades que les permita comercializar sus innovaciones.

A pesar de la relevancia que tiene para el desarrollo económico de las naciones la transferencia de tecnología, en este caso de patentes, entre la universidad y la industria, se han realizado pocos estudios sobre el tema en los países en desarrollo (Varzim y Rapini, 2010, en Closs et al., 2012).

Los resultados obtenidos confirman los señalamientos emitidos por De Gortari Rabiela (2002) sobre la escasa transferencia de conocimiento y desarrollo tecnológico que implica la necesidad de impulsar la función de comercialización de las innovaciones en los CC, desarrollando competencias en el personal y/o contratando personal ex profeso para ello. Propuesta que en el mismo sentido plantean Closs et al. (2012), al indicar que la transferencia de tecnología exige la creación de estructuras institucionales que permitan a los investigadores dedicarse a este proceso y un programa de formación para investigadores donde se desarrollen habilidades comerciales.

Además debería considerarse que algunas empresas necesitan innovar para mantener su competitividad, hecho que detona el trabajo cooperativo entre los CC y los sectores productivos, aunque ello implique el que los investigadores diseñen sus proyectos con el objetivo de dar respuesta a las necesidades tecnológicas de las empresas, lo que garantizaría la transferencia de las invenciones.

\section{CONCLUSIONES}

Las estadísticas mostradas permiten observar que la empresa mexicana no es favorecida por la propiedad industrial que tienen en su cartera de productos los CC. Sólo $8.82 \%$ de los títulos de propiedad industrial registrados en México ha sido transferido y el resto se conserva inactivo; es decir, no ha generado un proceso de licenciamiento o de venta de la patente al sector empresarial. Por ello es pertinente que la propiedad industrial que está lista para ser transferida encuentre su camino hacia los distintos sectores, de ahí la conveniencia de diseñar políticas públicas que detonen dicho proceso. 
Siguiendo a Calderón Martínez (2014: 38):

los derechos de propiedad industrial no se consideran únicamente un documento legal sino que, en algunos casos, están presentes en aspectos que van desde el intercambio de conocimiento hasta la obtención de fondos para la investigación, la protección y transferencia de resultados.

Ante un escenario de recortes presupuestales como el actual, la transferencia de patentes es un medio para la obtención de recursos financieros que les permitan a los CC seguir trabajando como hasta ahora.

Es importante señalar que el estudio se limitó a la transferencia de propiedad industrial por comercialización, la cual "se refiere a intercambios basados en actividades científicas con una orientación comercial sobre procesos o productos específicos: empresas de base científica, contratos de investigación, asesorías, patentes y licencias, entre otros" (Stezano Pérez, 2012: 14); en el futuro es importante ampliar el análisis e incluir la transferencia formal e informal mediante, por ejemplo, la clasificación establecida por Stezano Pérez (2012: 14), quien argumenta que

el canal informal incluye las interacciones personales establecidas sin que medien en ellas relaciones organizacionales formales, mientras que el canal formal implica la transmisión de conocimientos codificados por medios formales, como publicaciones, reportes y conferencias; y tácitos vía capital humano, tales como acuerdos organizacionales formales para la movilidad de personal y el entrenamiento de fuerza laboral.
Además del planteamiento de generar estrategias de política pública que incentiven la transferencia de títulos de propiedad industrial, se hace necesario llevar a cabo un análisis al interior de los CC que permita identificar fortalezas, debilidades, oportunidades y amenazas, y con ello realizar planteamientos específicos para aumentar los indicadores asociados a variables de licencias y patentes. Este análisis permitirá tener en cuenta variables adicionales que permitirían hacer un estudio más puntual, como puede ser la edad del investigador, el género, si cuenta con posdoctorado -nacional o internacional-, antigüedad en el CC, entre otras; así como revisar lo que se está haciendo en el sector privado y el papel que juegan las universidades en los mismos indicadores.

Finalmente, la información aquí vertida permite hacer un comparativo entre los indicadores referidos a títulos de propiedad industrial entre los CC, así como la situación que guardan con el sector privado; la importancia de los valores mostrados permite que estos sean tomados por los propios CC para tener un marco de referencia (benchmarking) y qué es lo que se tendría que hacer si el objetivo fuera mantenerse en la cima de la clasificación - avanzar hacia los primeros lugares. De la misma forma, se vuelve un elemento importante para las administraciones estatales, que pueden a su vez generar estrategias específicas (normativas, fiscales, financieras y alianzas) para que estos CC se vinculen con los sectores educativos y productivos locales; asimismo, se transformen en un motor de desarrollo empresarial con capacidad de atracción de empresas innovadoras que desarrollen productos nuevos y exista énfasis en la mejora de procesos.

\section{LITERATURA CITADA}

- Cabello, A. y ORTIZ, E. Políticas públicas de innovación tecnológica y desarrollo: teoría y propuesta de educación superior. Convergencia, 20(61): 135-172, 2013.

- CAlderón martínez, G. Patentes en Instituciones de Educación Superior en México. Revista de la Educación Superior, XLIII(2) (170): 37-56, 2014.

- CLOSS, L. et al. Intervenientes na Transferência de Tecnologia Universidade-Empresa: O Caso PUCRS. RAC-Revista De Administração Contemporânea, 16(1): 59-78, 2012.

- DAUTREY, P. Desarrollo y apertura económica en México: la eterna cuestión de los eslabones perdidos. RIPS Revista de Investigaciones Políticas y Sociológicas, 7(2): 153-166, 2008.
- DE GORTARI RABIELA, R. Impacto de la demanda empresarial en los centros de investigación y desarrollo. Nueva Antropología. Revista de Ciencias Sociales, número 60, 89-100, 2002.

- KANTIS, H. y DÍAZ, S. Estudio de Buenas Prácticas. Innovación y emprendimiento en Chile: una radiografía de los emprendedores dinámicos y de sus prácticas empresariales. Santiago, Chile: Endeavor, 2008.

- LOMELÍ VANEGAS, L. y MURAYAMA RENDÓN, C. México frente a la crisis: hacia un nuevo curso de desarrollo. Economía UNAM, 6(018): 7-60, 2009.

- LÓPEZ G., S. et al. Un acercamiento al concepto de la transferencia de tecnología en las universidades y sus diferentes manifestaciones. Panorama Socioeconómico, 24(32): 70-81, 2006. 
- OECD IORGANISATION FOR ECONOMIC CO-OPERATION AND DEVELOPMENT) OECD Patent Statistics Manual. Paris, France: Organisation for Economic Cooperation and Development, 2009.

- OMPI (ORGANIZACIÓN MUNDIAL DE LA PROPIEDAD INTELECTUAL) Principios básicos de la propiedad industrial. Publicación 895 (S). Suiza: Autor.

- SALVADOR BENÍTEZ, J. L. Desarrollo, educación y pobreza en México. Papeles de población, 14(55): 237-257, 2008.

- STEZANO PÉREZ, F. A. Construcción de redes de transferencia ciencia-industria en el sector de biotecnología en México: Estudio de caso sobre las vinculaciones tecnológicas entre investigadores de CINVESTAV Irapuato y LANGEBIO y empresas del sector agro-biotecnológico. Estudios sociales, 20(39): 9-38, 2012.

\section{De páginas electrónicas}

- CÁmARA DE DIPUTADOS. Ley de ciencia y tecnología. Diario Oficial de la Federación, 5 de junio de 2002. Recuperado el 5 de abril de 2016, de http://www.diputados.gob.mx/LeyesBiblio/ pdf/242_081215.pdf

- CÁMARA DE DIPUTADOS. Ley Orgánica del Consejo Nacional de Ciencia y Tecnología. Diario Oficial de la Federación, 20 de mayo de 2014. Recuperado de http://www.diputados.gob.mx/ LeyesBiblio/pdf/243.pdf

- conacyt (CONSEJO NACIONAl DE CIENCIA y teCNOlOgía) Anexos informe 2013. Anexo capítulo II. Recursos humanos en ciencia y tecnología. México: Autor. 2010-2013. Recuperado de http://www.siicyt.gob.mx/index.php/estadisticas/informegeneral/informe-general-2013/anexos-informe-2013,

http://www.siicyt.gob.mx/index.php/estadisticas/informegeneral/informe-general-2010,

http://www.siicyt.gob.mx/index.php/estadisticas/informegeneral/informe-general-2011,

http://www.siicyt.gob.mx/index.php/estadisticas/informegeneral/informe-general-2012,

http://www.siicyt.gob.mx/index.php/estadisticas/informegeneral/informe-general-2013

- conacyt (CONSEJO NACIONAL DE CIENCIA y teCNOLOGÍA) Anuarios estadísticos 2001-2009, SIICYT. [Portal] México: Autor, s.f. Recuperado de http://www.siicyt.gob.mx/index.php/ estadisticas/informe-general

- CONACYT (CONSEJO NACIONAL DE CIENCIA y TECNOLOGÍA) Centros públicos de investigación Conacyt, Conacyt. 2014. Recuperado el 4 de marzo de 2014, de http://conacyt.gob.mx/ index.php/el-conacyt/centros-de-investigacion-conacyt

- conacyt (CONSEJO NACIONAL DE CIENCIA y TECNOLOGÍA) Comunicado de prensa 38/11: Urgen a incrementar el número de patentes en el país. D. F., México: Autor, 26 de abril de 2011. Recuperado el 4 de marzo de 2014, de http://2006-2012.conacyt. gob.mx/comunicacion/comunicados/Paginas/38-11.aspx

- conacyt (CONSEjo nacional de Ciencia y teCnOlOgía) Evalúan desempeño de los centros públicos CONACYT. Veracruz, México: Autor, 27 de abril de 2010. Recuperado el 24 de noviembre de 2016, de http://2006-2012.conacyt.gob.mx/ comunicacion/comunicados/Paginas/30-10.aspx

- CONACyt (CONSEJO NACIONAL DE CIENCIA Y TECNOLOGÍA) Programa Especial de Ciencia y Tecnología 2008-2012. Diario Oficial de la Federación, 16 de diciembre de 2008. Recuperado el 24 de noviembre de 2008, de http://dof.gob.mx/nota_detalle. php? codigo $=5074624 \&$ fecha $=16 / 12 / 2008$

- DUTTA, S. y LANVIN, B. (Eds.). The global innovation index 2013. The local dynamics of innovation. Ithaca-Fontainebleau-Geneva: Cornell University-INSEAD-WIPO. Recuperado el 10 de marzo de 2014, de http://www.wipo.int/edocs/pubdocs/en/economics/ gii/gii_2013.pdf

- IMPI (INSTITUTO MEXICANO DE LA PROPIEDAD INDUSTRIAL) IMPI en Cifras 2014. México: Autor. 2014. Recuperado el 10 de marzo de 2014, de http://www.impi.gob.mx/QuienesSomos/ICIFRAS/ IMPI_CIFRAS_ene_sep_2013.pdf

- INEGI (INSTITUTO NACIONAL DE ESTADÍSTICA Y GEOGRAFÍA) Encuesta sobre Investigación y Desarrollo Tecnológico (2010). México: Autor, s. f.a. Recuperado el 5 de junio de 2014, de http://www.inegi.org.mx/est/contenidos/proyectos/ accesomicrodatos/esidet/default.aspx

- INEGI (INSTITUTO NACIONAL DE ESTADÍSTICA Y GEOGRAFíA) Sistema de cuentas nacionales. México: Autor, s. f.b. Recuperado el 5 de junio de 2014, de http://www.inegi.org.mx/est/contenidos/ proyectos/scn/

- OECD IORGANISATION FOR ECONOMIC COOPERATION AND DEVELOPMENT) Main science and technology indicators 2011/2. Paris, France: OECD, 2012. Recuperado el 8 de marzo de 2014 , de http://www.oecd.org/science/inno/49501885.pdf

- OCDE (ORGANIZACIÓN DE COOPERACIÓN Y DESARROLLO ECONÓMICOS)-EUROSTAT (OFICINA DE ESTADÍSTICAS DE LAS COMUNIDADES EUROPEAS) Manual de Oslo. Guía para la recogida e interpretación de datos sobre innovación. 3 ed. Autor, 2005. Recuperado el 2 de junio de 2016, de http://www.dgi.ubiobio.cl/ dgi/wp-content/uploads/2010/07/manualdeoslo.pdf

- SECOFI (SECRETARÍA DE COMERCIO Y FOMENTO INDUSTRIAL). Ley de Fomento y Protección de la Propiedad Industrial. Diario Oficial de la Federación, 27 de junio de 1991. Recuperado el 3 de mayo de 2014, de http://dof.gob.mx/index. php? year $=1991 \&$ month $=06 \&$ day $=27$

- SHCP (SECRETARÍA DE HACIENDA Y CRÉDITO PÚBLICO) Cuenta pública. México: Autor, s. f. Recuperado el 6 de septiembre de 2014, de http://www.shcp.gob.mx/EGRESOS/contabilidad_ gubernamental/Paginas/cuenta_publica.aspx

- VILLALOBOS GARCíA, L. y PONCE TALANCÓN, H. La educación como factor del desarrollo integral socioeconómico. Contribuciones a las Ciencias Sociales, julio de 2008. Recuperado de http://www.eumed.net/rev/cccss/02/vgpt.htm

- WIPO (WORLD INTELLECTUAL PROPERTY ORGANIZATION) World Intelectual Property Indicators. Economic \& Statistic Series. Switzerland: WIPO, 2013. Recuperado el 10 de marzo de 2014 , de http://www.wipo.int/edocs/pubdocs/en/intproperty/941/ wipo_pub_941_2013.pdf 